\title{
EXAMINING RISK COMMUNICATION OF SAMSUNG BY ANALYSING CUSTOMER REACTION ON SOCIAL MEDIA: FROM GALAXY NOTE 7 EXPLOSION TO GALAXY S8 RELEASE
}

\author{
Jaewon Lim and Jang Hyun Kim \\ Department of Interaction Science, Sungkyunkwan University, Seoul, Korea
}

\begin{abstract}
Crisis of product defect causes recalls, which not only causes financial loss but also devastates brand reputation. This study explores customers' response to the twice recall of the Galaxy Note 7 in Korea. The authors examined the semantic networks of consumers comments from the Samsung official social media 'Samsung Newsroom (blog and Facebook)' in five time periods(i.e., release, first recall, recommendation to discontinue use, second recall, and compensation program). Customers in each period showed different emotional states which were positive in the first recall but negative in the second recall. Therefore, timely recall and factual communication is needed to the product-harm crisis management, but root cause investigation should be taken to avoid further recalls.
\end{abstract}

\section{KEYWORDS}

Galaxy Note7, Product harm crisis, Recall, Semantic network analysis

\section{INTRODUCTION}

The crisis of the Galaxy note 7 recall occurred within a month of its release. Galaxy note 7 was unveiled on August 19, 2016. However, several Galaxy note 7 explosion cases were reported and the epidemic was spreading. Samsung diagnosed the cause of the explosion is a failure of the batteries and recalled the Note 7. Although they provided replacement phones, those began to explode as well. In the end, Samsung issued the second recall and recalled the entire line of Note 7 phones.

[1]Recalling a product can make the involved firms' brand image worse.[2]Therefore, they should try to reduce negative impacts, one of which is to convey their official position to the public through media.

[3] Since the mid-2000s, Social media platforms increasingly tend to act as both a place for the firms and customers. Focal firms' risk communication message can be delivered instantly and broadly. With social media, customers can voice their opinion. Samsung posted a statement in Natarajan Meghanathan et al. (Eds) : CCSEA, NCOM, AIFU, DKMP, EMSA, SIPRO, SEA - 2018 pp. 63-71, 2018. () CS \& IT-CSCP 2018 
their official social media channel, 'Samsung Newsroom(blog and Facebook)' during a series of explosion event. At the same time, the customers commented on each statement to voice their opinion.

[4]As far as the authors investigated, Samsung is perhaps the only company to recall twice and has the largest recall in terms of volume. However, as customers become more active and social network service develops, product recalls may happen more frequently in the future. By assessing Samsung's crisis management to the Galaxy note 7, this study aims to understand the successful product crisis management. Using the semantic network analysis, the authors analyzed the customers' comments written under the Samsung official announcements in their social channel. Therefore, we proposed the following research questions :

RQ1: How customers react to Samsung's risk communication on social media(blog and Facebook)?

RQ1-1. What are semantic traits of the blog and Facebook comments?

RQ1-2. What are common and unique words for each period?

RQ1-3. How did emotional words change over time?

\section{METHOD}

Figure 1 shows the overall research process.

\begin{tabular}{|c|c|c|}
\hline Select data collection period & Data preprocessing & Semantic network analysis \\
\hline $\begin{array}{l}\text { Selecting and dividing time } \\
\text { period by Samsung's official } \\
\text { announcement for each issue } \\
\text { point }\end{array}$ & \multirow{3}{*}{$\begin{array}{l}\text { 1. Data consolidation for } \\
\text { colleting, selecting and } \\
\text { integrating data } \\
\text { 2. Data cleaning for imputing } \\
\text { missing value, reducing noise } \\
\text { in data and eliminating } \\
\text { inconsistencies } \\
\text { 3. Data transformation for } \\
\text { normalizing, aggregating data } \\
\text { and constructing new attributes } \\
\text { 4. Data reduction for reducing } \\
\text { number of words } \\
\text { 5. Well-formed data }\end{array}$} & \multirow[t]{3}{*}{$\begin{array}{l}\text { 1. Words clustering } \\
\text { 2. Words categorizing } \\
\text { 3. Visualization }\end{array}$} \\
\hline Data collection & & \\
\hline $\begin{array}{l}\text { Collecting comments data } \\
\text { from Samsung official social } \\
\text { media channel 'Samsung } \\
\text { newsroom(blog and } \\
\text { facebook)' }\end{array}$ & & \\
\hline
\end{tabular}

Figure 1. Overall research process

\subsection{Select data collection period}

The authors estimated the change of the word by period and analyze the change of customers' awareness about the issue. The data collection period was divided into six periods. The first data collection period is from August 11, 2016 to August 17. It covers six days from the product launch. The second data collection period is September 2, 2016, when Samsung voluntarily announced the recall. The third data collection period is from September 10, 2016 to September 20, 2016. During this period, Samsung advised their users to discontinue the use of the Galaxy Note7 and announced instructions for commencing the exchange of products. The fourth data 
collection period is October 11, 2016, when Samsung officially announced the discontinuation of Galaxy Note7. The fifth data collection period is October 13, 2016 to October 27. It was time for Samsung to announce compensation program, called 'Galaxy upgrade program', including exchange and refund. Last period is April 19, 2017, when Galaxy s8 released. Galaxy s8 is the first and new Samsung galaxy series product after the Galaxy Note 7 explosion.

\subsection{Data Collection}

Customer comments were gathered from the Samsung's official social media channel 'Samsung Newsroom (blog \& Facebook)'. We scraped all the comments manually. A total of 4,016 comments were collected during the data collection period. All of the comments are in Korean as the authors examined only the newsroom for Koreans.

Only Galaxy s8 customer comments were gathered from six news articles posted in the Naver.

\subsection{Data preprocessing}

Text preprocessing was carried out through Textom, an online text analysis service which is a Korea-based big data processing solution site.

The authors uploaded the collected text data to Textom and preprocessed. In this process, the main words were identified through the frequency of extracted words. Basically, it provides listing of nouns, adjectives, and verb phrases that remove special letters and postposition through morphological analysis. Words with similar meanings are grouped together into represented word. In this way, only the meaningful words were selected from the word list formed by refining and the co-occurrence frequency matrix data was produced.

\subsection{Semantic Network Analysis}

[5]Semantic network analysis (SNA) was conducted, which examines the relationships among words such as co-occurrence, frequency, and distance. As SNA adequately shows the significant words regarding the customers opinion and emotions about Samsung's product crisis management, it is opted to employ for the present study.

[6]To visualize the relationship between words, we used computational text analysis tools NETDRAW from UCINET6.

Among a variety of centrality measures, betweenness centrality was selected for this study.

\section{RESULTS}

To visualize semantic traits of words, the authors categorized words into four groups. The words which are related to facts of issue were categorized into group 'Fact' with black nodes. Also, emotional words were considered for grouping. Positive words were specified 'Positive feeling' with blue nodes and negative words were grouped into 'Negative feeling' with red nodes. Finally, words about cost were categorized into 'Cost' with yellow nodes. 


\subsection{Six days from the product launch- August 11, 2016 to August 17, 2016}

For the first period, 73 comments were collected. The words with the highest frequency next to 'Samsung' are article, opening, Galaxy Note7, good, consultation, head office, change and others.

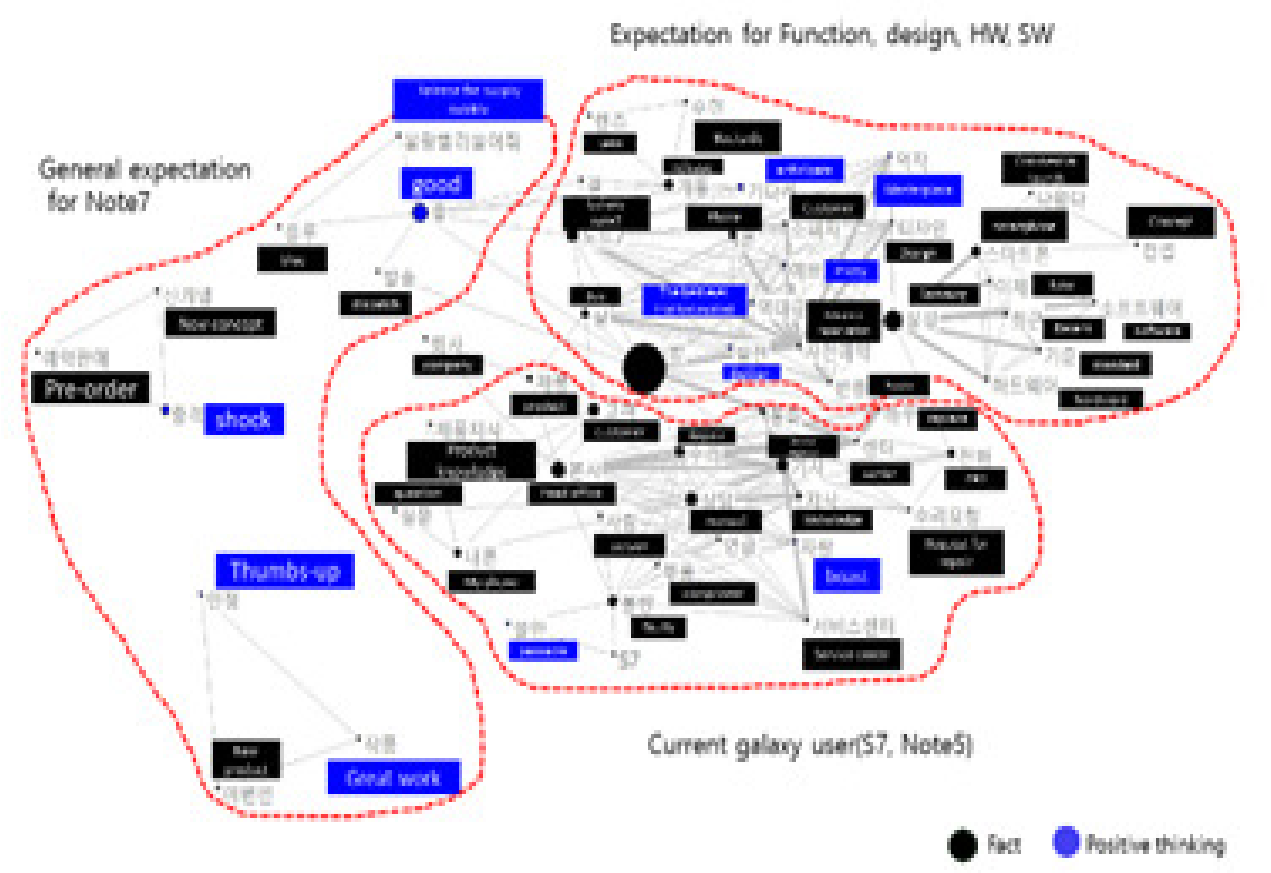

Figure 2. Semantic network of comments on Samsung Newsroom from August 11, 2016 to 17

In Figure2, words are related to 'Current Galaxy users (S7, Note5)' and 'General expectation for Note7'. For example, words explaining current Galaxy users are product, service center, repair, and Galaxy S7. Such words as design, software, hardware, pre-order, lens and better were mentioned for general expectation for Note7. In this period, most words are categorized into group 'Fact' and 'Positive feelings'.

\subsection{Recall announcement - September 2, 2016}

For the second period, 462 comments were collected. The words with the highest frequency next to 'Samsung' are product, exchange, decision, battery, refund, pre-order, recall, new product, problem, use, customer, corporation, and others.

In Figure3, words are related to two topics, which are 'Explosion issue and disappointed feeling' and 'Positive opinion for Samsung decision.' For example, words about the first topic are explosion, tiresome, disappointment, problem, defect, bomb, return, pity, and error. For the second topic, good decision, trust, thanks, and cheer up were mentioned.

At this time, lots of words are categorized into group 'Fact' and 'Positive feelings' like the first period semantic network. Also, words with 'Negative feelings' were salient from this point. 


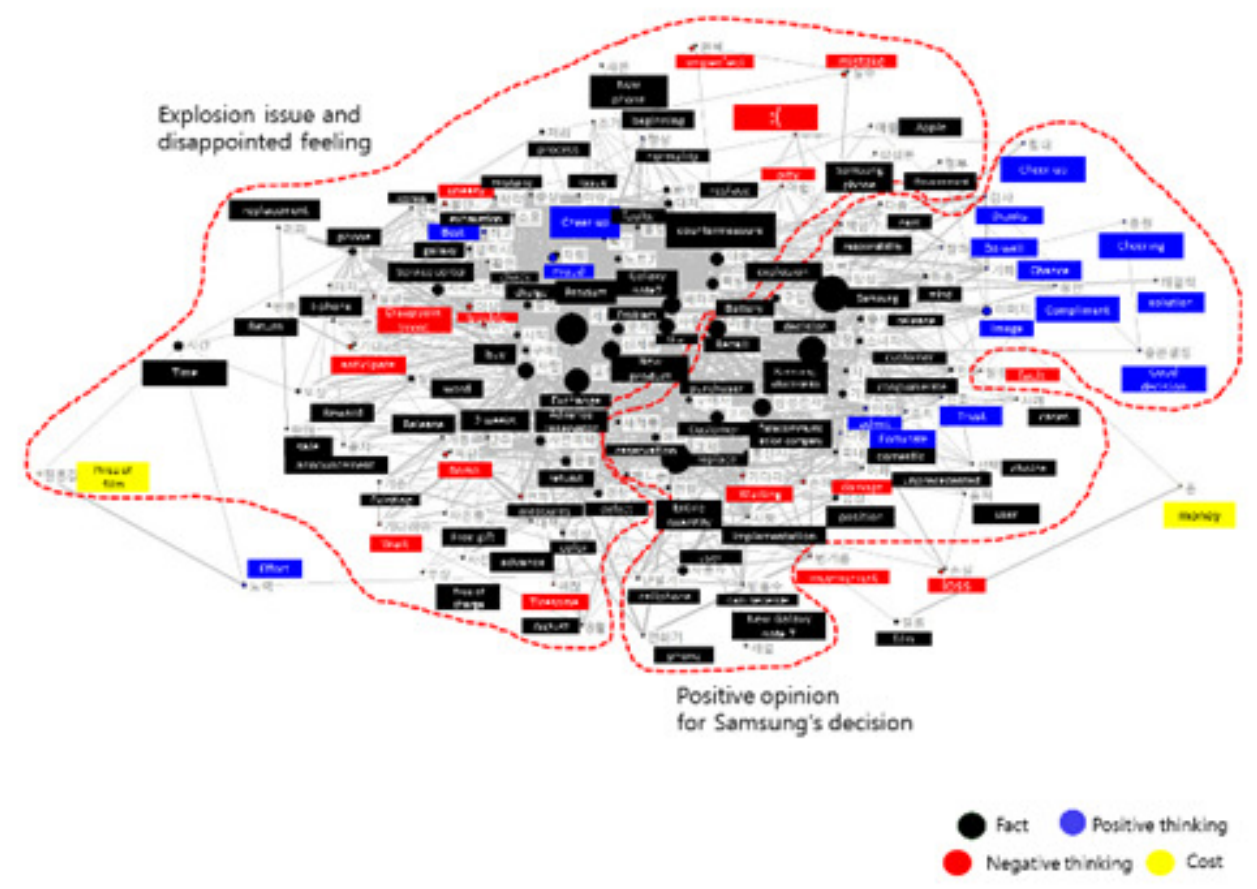

Figure 3. Semantic network of comments on Samsung Newsroom on September 2, 2016

\subsection{From recommendation for stop using to exchanging replacement - September 10, 2016 to September 20, 2016}

For the third period, 515 comments were collected. The most frequent words next to 'Samsung' are exchange, customer, battery, product, problem, wait, Galaxy Note7, authorized dealer, explosion, reservation, recall, purchase and other words.

In Figure4, words described 'Explosion issue and product problems' and 'Complaint for exchanging and disappointment.' For example, the words related to explosion issue and product problems are explosion, fault, dead battery, defect, and safety. For the complaint for exchanging and disappointment, the words such as it's annoying, inconvenience, refusal and apology were mentioned.

During this period, while blue nodes with 'Positive feeling' decreased sharply compare to previous two periods, red nodes with 'Negative feeling' increased.

\subsection{Second recall - October 11, 2016}

For the fourth period, 1,002 comments were collected. Word 'Exchange' is the most frequently mentioned at this time. Refund, Samsung, Galaxy Note7, customer, purchase, use, think, product, compensation, change, wait, and cancellationcharge are the highest frequency words with 'Exchange' 


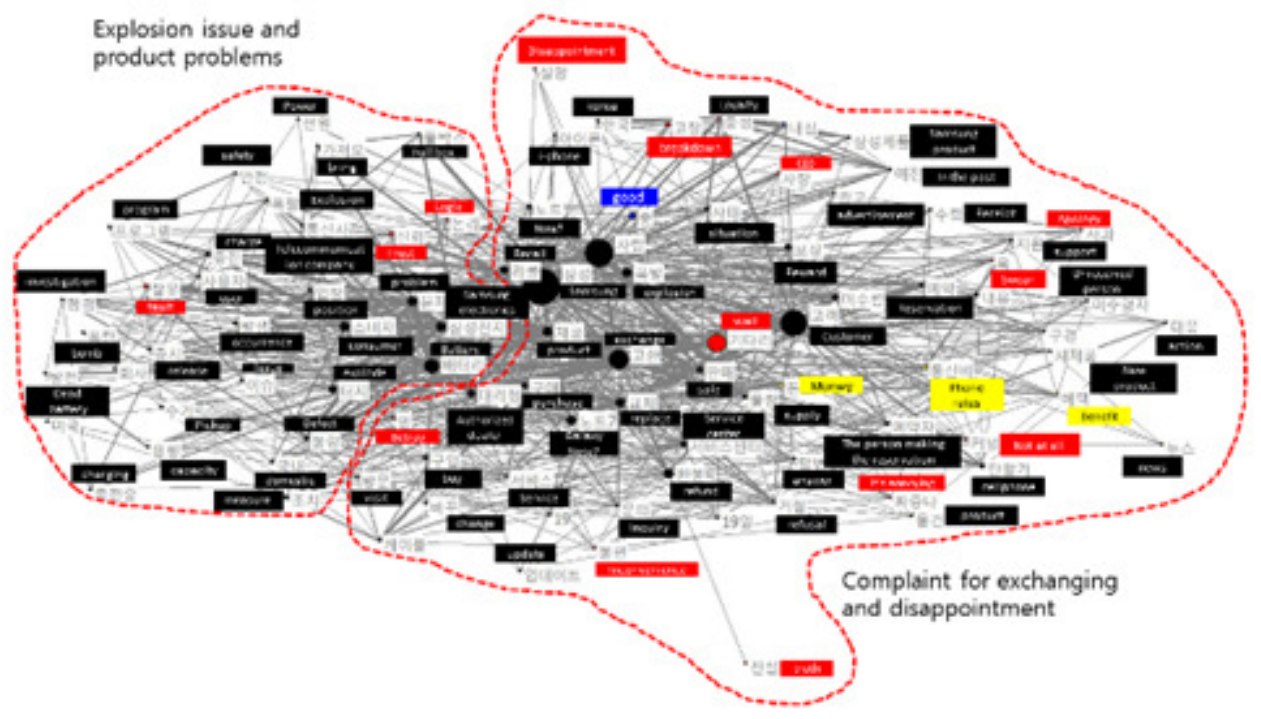

Fact Positive thinking

Negatve thiniang Cost

Figure 4. Semantic network of comments on Samsung Newsroom from September 10, 2016 to September 20,2016

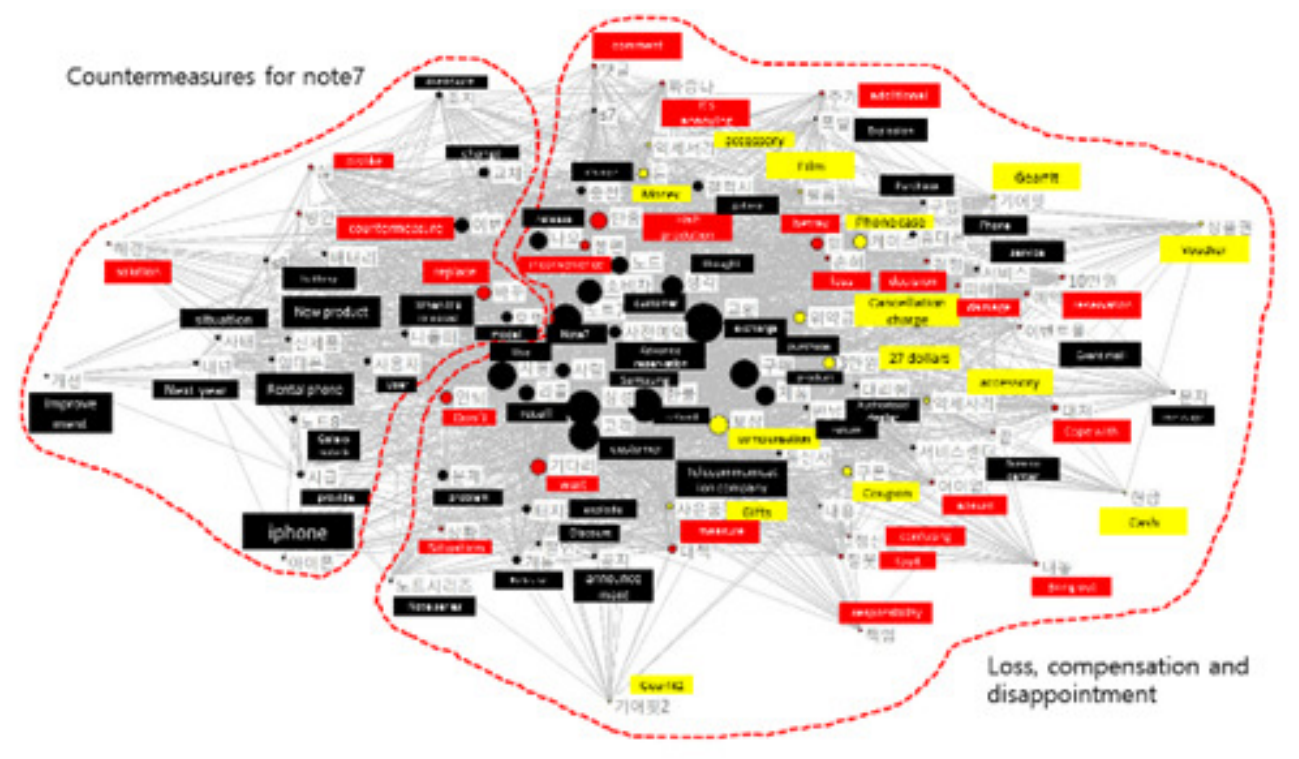

Fact Positive thinking
Negative thinking Cost

Figure 5. Semantic network of comments on Samsung Newsroom on October 11, 2016 
In Figure5, words are demonstrating two topics, which are 'Loss, compensation and disappointment' and 'Countermeasures for Galaxy Note7.' For example, the words regarding the first topic are cancellation, charge, damage, halt production, absurd, and compensation. For the second topic, Galaxy Note8, Galaxy S8, iPhone, new product, and rental phone were mentioned.

At this time, lots of words are categorized into group 'Cost' and 'Negative feeling' unlike other three periods.

\subsection{Compensation program notice - October 13, 2016 to October 27, 2016}

For the last period, 2,022 comments were collected. The most frequent words next to 'Samsung' are exchange, customer, refund, compensation, opinion, Galaxy Note7, use, change, product, program, purchase, return, Galaxy Note8, and others.

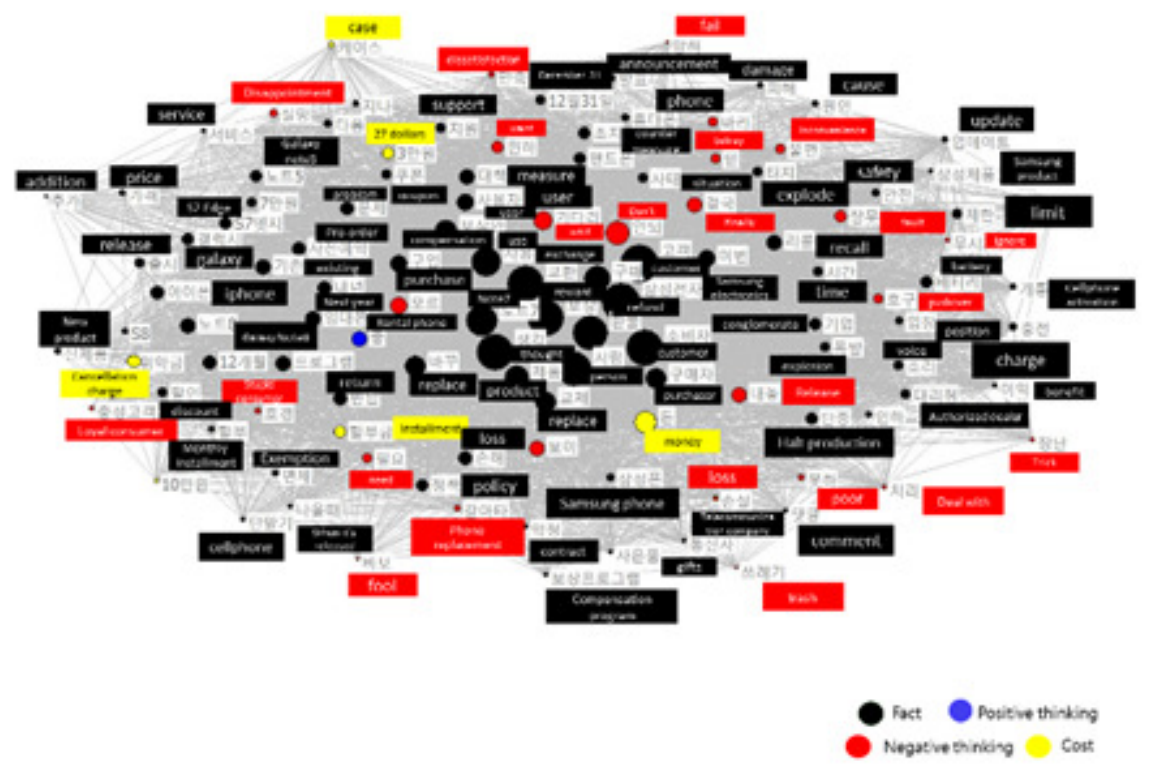

Figure 6. Semantic network of comments on Samsung Newsroom from October 13, 2016 to October 27, 2016

In Figure6, most words in categories 'Fact', 'Negative feeling', and 'Cost' are mentioned similarly. Black, red and yellow nodes are spotted sporadically. For example, words like money, installment, (smartphone) case, 30 dollars, and coupon are showing yellow nodes 'Cost'. Dissatisfaction, trash, fool, wait, fail, discomfort, phone replacement, and pushover present red nodes 'Negative feeling'. This network has only one blue node for 'Positive feeling' with word 'good'.

\subsection{Galaxy s8 release}

We can see the unique word 'Sakura Edition'. Despite having to be cautious after the battery explosion of the Galaxy Note 7, the phenomenon that the display screen is reddish immediately after the launch of the Galaxy S8 was controversial. It got a humiliating nickname of'Sakura Edition', and consumers blamed it as 'Trash Galaxy' and kicked the tongue. In addition, it is the 
first new product after the explosion, so consumers' worries about 'Safety' have increased. The words 'Note 7', 'Battery' and 'Explosion' related to Note 7 were also mentioned.

Although there are few numbers, positive words also appeared. A few consumers were satisfied and admitted with the design of the Galaxy S8 and cheered on Samsung('Fighting'

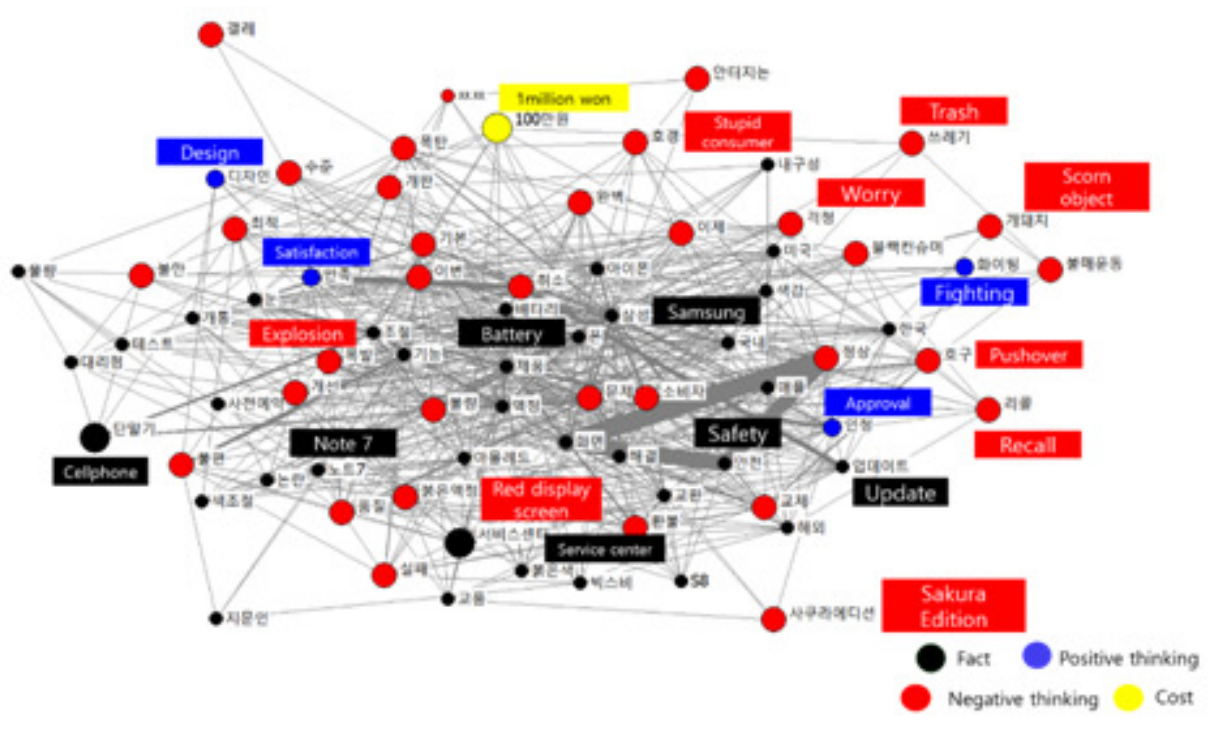

Figure 7.Semantic network of comments on Galaxy s8

\section{DISCUSSION}

As a result of examining the crisis response of Samsung in five periods as above, it can be seen that Samsung's product crisis management on the Galaxy note 7 was not so effective as desired. It was a good decision to admit the product defect and to make a swift recall. As we can see the words in the second period, people felt "trust" in Samsung's recall. However, due to the rapid exchange of products without properly identifying the cause, replacements also exploded. This led to second recalls and customers had to do "tiresome" exchanges even twice. Customers even need to seek a smartphone to replace the Galaxy Note 7 and to use 'iPhone' or Galaxy s7 edge which is older than Galaxy Note 7.

When Samsung announced the first recall, it would have been possible to reduce the damage if they had enough time to properly identify the cause. It is a smart move to admit product defects in detail and voluntarily recall products as early as possible if the cause of the defect is the problem of the product itself. Without uncovering the root cause, rashness can result in the two times of recalls like Galaxy note 7 . Not only timely but accurate and factual communication is needed.

Second, results show that the importance of an appropriate compensation program. After Samsung announced that it has halted sales and production of the Galaxy Note 7 worldwide, Samsung is offering a new compensation program. However, it is not much different from the existing 'Galaxy program', which was packaged as if it were a special reward program for Galaxy note 7 customers. So when the authors analyzed the comments, the word 'trickery' appeared frequently. It should be noted that anger that is worse than a product defect came from the users 
feeling that the company may have deceived its customers.

Third, the performance of the device in launching a new product after the crisis must be emphasized. For the new products released after the crisis, consumers expressed their negative opinions due to the previous explosion (the 'red display screen' phenomenon of the Galaxy S8 also contributed to the negative comments), but at the same time it showed a positive response to the 'performance' and 'design' of the s8.Therefore, when it comes to product launches in the future, it will be more important to improve the 'performance' and 'safety' of products.

\section{CONCLUSION AND LIMITATION}

[7]Samsung sits at 49th place in 2017 Reputation Quotient Ratings, which ranks one of American's 100 most visible companies. This is comparable to the 7th rank in 2016 and all three consecutive years when Samsung secured a spot in the top 10 from 2014 to 2016.

The limitation of this study is in that it explored comments from Korean Facebook and blog, although Galaxy note7 recall occurred worldwide.

\section{REFERENCES}

[1] LAUFER, Daniel; COOMBS, W. Timothy. How should a company respond to a product harm crisis? The role of corporate reputation and consumer-based cues. Business Horizons, 2006, 49.5:379-38

[2] J. HOLMES, Bev, et al. Communicating with the public during health crises: experts' experiences and opinions. Journal of Risk Research, 2009, 12.6: 793-807.

[3] RUTSAERT, Pieter, et al. The use of social media in food risk and benefit communication. Trends in Food Science \& Technology, 2013, 30.1: 84-91.

[4] Aditya Madanapalle. (2016) Samsung Galaxy Note 7 recall: Here's a look at a few device recalls from the past. http://www.firstpost.com/tech/news-analysis/samsung-galaxy-note-7-recall-her es-a-look-ata-few-device-recalls-from-the-past-3688793.html (accessed Oct.18, 2017)

[5] LEE, Sungjoon; HYUN KIM, Jang; ROSEN, Devan. A semantic network and categorical content analysis of Internet and online media research. The Open Communication Journal, 2009, 3.1.

[6] BORGATTI, Stephen P.; EVERETT, Martin G.; FREEMAN, Linton C. Ucinet for Windows: Software for social network analysis. 2002.

[7] The Harris Poll. (2017) 2017 Reputation Quotient Ratings. http://www.theharrispoll.com/reputationquotient (accessed Oct.18, 2017)

\section{AUTHORS}

Jaewon Lim is studying Interaction and Science in Sungkyunkwan University. Main research interests lie in social and semantic network analyses and media and communication.

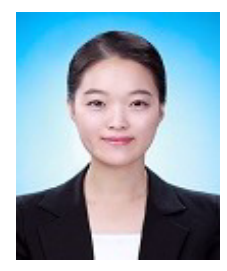

